\title{
RESEARCH
}

Open Access

\section{Adding patient-reported outcomes to a multisite registry to quantify quality of life and experiences of disease and treatment for youth with juvenile idiopathic arthritis}

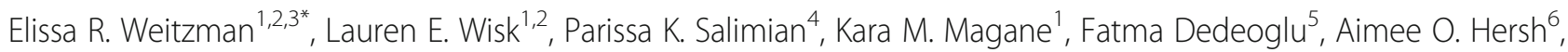
Yukiko Kimura ${ }^{7}$, Kenneth D. Mandl ${ }^{2,3,8}$, Sarah Ringold ${ }^{9}$ and Marc Natter ${ }^{2,3}$

\begin{abstract}
Background: Children with Juvenile Idiopathic Arthritis (JIA) often have poor health-related quality of life (HRQOL) despite advances in treatment. Patient-centered research may shed light on how patient experiences of treatment and disease contribute to $H R Q O L$, pinpointing directions for improving care and enhancing outcomes.

Methods: Parent proxies of youth enrolled in the Childhood Arthritis and Rheumatology Research Alliance (CARRA) Registry shared patient-reported outcomes about their child's HRQOL and experiences of disease and treatment burden (pain interference, morning stiffness, history of medication side effects and methotrexate intolerance). Contributions of these measures to HRQOL were estimated using generalized estimating equations accounting for site and patient demographics.

Results: Patients $(N=180)$ were $81.1 \%$ white non-Hispanic and 76.7\% female. Mean age was $11.8(S D=3.6)$ years, mean disease duration was 7.7 years ( $S D=3.5)$. Mean Total Pediatric Quality of Life was $76.7(S D=18.2)$. Mean pain interference score was 50.1 ( $S D=11.1$ ). Nearly one-in-five $(17.8 \%)$ youth experienced $>15$ min of morning stiffness on a typical day, more than one quarter (26.7\%) reported $\geq 1$ serious medication side effect and among 90 methotrexate users, $42.2 \%$ met criteria for methotrexate intolerance. Measures of disease and treatment burden were independently negatively associated with HRQOL (all $p$-values $<0.01$ ). Negative associations among measures of treatment burden and HRQOL were attenuated after controlling for disease burden and clinical characteristics but remained significant.

Conclusions: For youth with JIA, HRQOL is multidimensional, reflecting disease as well as treatment factors. Adverse treatment experiences undermine HRQOL even after accounting for disease symptoms and disease activity and should be assessed routinely to improve wellbeing.
\end{abstract}

Keywords: Health-related quality of life, Disease burden, Treatment burden, Patient reported outcomes, Juvenile idiopathic arthritis, Children, Adolescents, Chronic illness

\footnotetext{
* Correspondence: Elissa.Weitzman@childrens.harvard.edu

${ }^{1}$ Division of Adolescent/Young Adult Medicine, Boston Children's Hospital,

300 Longwood Ave, Boston, MA 02115, USA

2Department of Pediatrics, Harvard Medical School, Boston 02115, USA

Full list of author information is available at the end of the article
} 


\section{Background}

Children with Juvenile Idiopathic Arthritis (JIA) face significant hurdles regarding health-related quality of life (HRQOL) related to the chronic relapsing nature of the condition, unpredictable disease course and difficult treatments [1-3]. Affected youth report poorer HRQOL than their peers even in the setting of low disease activity and treatment with biologic disease modifying agents [4-6]. Nearly half of children with JIA have recurrent or ongoing disease activity on entry into adulthood, with active arthritis, progressive joint damage, exposure to chronic arthritis treatments and decreased HRQOL [5, 7-10].

For youth with JIA, experiences of disease symptoms and disease activity negatively impact HRQOL [11]. There is also potential for medication side effects and negative treatment experiences (e.g. repeated intravenous infusion, frequent injections) to cause pain and stress, undermining wellbeing. Although greatly improved treatments are available for youth with JIA, many treated youth continue to suffer from poor HRQOL. Efforts to advance treatment acceptability, efficacy and HRQOL may hinge on a more mature evidence base. Patient-centered outcomes research (PCOR) provides a galvanizing framework to advance the evidence base by investigating the broad set of factors-disease and treatment-related-that contribute to wellbeing, with emphasis on concerns prioritized by patients $[11,12]$. PCOR may be especially informative for fostering evidence to improve the care of youth with JIA - their experiences of disease and treatment may differ from the perceptions of others including clinicians $[13,14]$. For youth, elucidating the specific and simultaneously estimated contributions to HRQOL of patient-centered and -reported measures of disease symptoms, disease activity, and difficulties with treatment may improve the acceptability and efficacy of care-identifying potentially ameliorable threats to wellbeing across a multifactorial model.

Where underlying conditions are uncommon or rare, as with JIA, disease registries may shed light on clinical factors and disease features relevant to understanding a condition and its course to support wellbeing $[15,16]$. For The Learning Cohort (TLC) study, we extended the registry model, leveraging the scalable informatics infrastructure constructed for the Childhood Arthritis and Rheumatology Research Alliance (CARRA) Registry [17-19], so as to enable Registry members with a diagnosis of JIA and their parent proxies to contribute Patient Reported Outcomes (PROs) for integration with Registry data. Following the PCOR approach, we elicited parents' leading concerns regarding treatment and hypothesized that leading concerns would be issues of medication safety/side effects. We further hypothesized that experience of treatment side effects and problems would adversely affect HRQOL after adjusting for the effects of disease symptoms and disease activity. Such findings, if confirmed, could powerfully inform the design and implementation of future PCOR and direct comparative effectiveness research (CER) toward addressing treatment concerns voiced by patients.

\section{Methods \\ Overview}

For the TLC study, children/adolescents with JIA enrolled in the CARRA Registry and their parents were engaged in completing structured PROs during routine care visits for the child. The overarching aim was to test a model of augmenting clinical registry data with PROs to improve understanding of the contributions to wellbeing of measures of disease and treatment burden. Participants were recruited using a convenience sampling strategy in which CARRA enrollees were approached for entry into a related cohort investigation that involved collection of PROs to complement Registry data; TLC recruiting occurred during routine clinic visits, from March 2014 to February 2016. Parents of patient participants completed all PRO measures about their child/ adolescent using parent proxy versions of PRO measures. Measures were programmed in the Research Electronic Data Capture (REDCap) system [20], from whence they could flow into the Registry using a modular, ontology-based, federated informatics infrastructure constructed from open source software; this infrastructure provides research investigators full ownership and access to their contributed data while supporting permissioned and robust data sharing across federated sites [17]. Upon enrolling in $T L C$, parents completed an electronic survey about their information needs and treatment concerns, their confidence and satisfaction with their child's care, their child's current use of medications, and their child's experiences of problems/side effects. Written informed consent/assent was obtained from all parent/child participants included in the study. No compensation was provided.

\section{Sites}

Four CARRA Registry clinical sites took part in this study: Boston Children's Hospital, Seattle Children's Hospital, Joseph M. Sanzari Children's Hospital at Hackensack University Medical Center, and University of Utah/Primary Children's Hospital. The research team at Boston Children's Hospital served as the coordinating site. The Institutional Review Boards of each participating institution approved the study.

\section{Participant eligibility}

Screening for eligibility was undertaken by an experienced clinical research coordinator $(\mathrm{CRC})$ at each site who reviewed medical record and Registry data prior to 
approaching patients. Patients and their parents were eligible if patients were enrolled in CARRA, younger than 18 years of age, had been diagnosed with JIA by a rheumatologist, and were taking prescription medication for their condition. Potential participants were ineligible if they were unable to speak and read English or use a tablet computer.

\section{Participation}

In all, 300 parent-patient dyads were approached for this study, of which 203 consented (67.7\% consent rate) and 191 contributed some form of data; of these, 11 were missing all PROs and were dropped from analyses, leaving a final analytic sample of 180 dyads.

\section{Measures}

Study domains were selected or developed with the input of experienced pediatric rheumatologists. Sources of data include: the CARRA Registry, the baseline parent survey, parent proxy reports of child: HRQOL and measures of disease and treatment burden. Additional file 1: Table S1 includes a summary of primary measures used.

\section{Demographic and clinical characteristics of patients}

Parents reported their child's age, sex, and race/ethnicity, as well as the highest education attained by a parent of the child as a proxy for socioeconomic status. For four subjects missing reports about parents' education, the sample mode was used. Age at onset of disease symptoms was obtained from the CARRA Registry, which was utilized to calculate disease duration by subtracting age of disease onset from age at survey administration. For three subjects missing data on disease duration, an age-based mean was used. Level of disease activity was also obtained from the Registry per the Physician Global Assessment (PGA) [21]. PGA is assessed on a $0-10$ scale, with 0 representing "not active" disease, 10 reflecting "very active" disease. The child's overall health status was reported by parents in response to the question: "When you consider all the ways your child's condition affects his/her life, how do you think your child is doing overall?" Parents selected a value between 1 and 10, with 1 being very poorly, 10 being very well.

\section{Medications}

Parents reported the child's current use of specific disease modifying anti-rheumatic drug (DMARD), biologic, and nonsteroidal anti-inflammatory drug (NSAID) medications taken for their JIA, selecting from medication lists grouped by category; they could also report "I don't know" or "Not on any of the above medications." Parents were also asked about their child's use of glucocorticoids (oral, intravenous, or local treatment including joint injections and eye drops) and herbal/non-vitamin supplements, for which response options were "yes", "no", and "I don't know." Parents could also enter any other medications their child takes for JIA in an open field.

\section{Parent reports about child's JIA treatment}

Parents reported the frequency of their child's rheumatology care visits, and their satisfaction with this care. Satisfaction was reported on a 4-point Likert scale ranging from "very satisfied" to "very dissatisfied."

\section{Leading concerns regarding child's treatment}

Parents were shown the following question: "When making decisions about your child's health, how concerned are you about the following issues related to prescription medications your child is taking?" and asked to select their top two concerns from a list of issues that spanned concerns with drug formulation, the potential for related psychosocial distress, immediacy/latency of harm. The list of items, which was informed by input from experienced pediatric rheumatologists' who drew on their clinical experiences of the most commonly voiced family concerns, included: correct dose and schedule of medication, safety of medication, serious short-term side effects (happen within a few days or weeks), most likely short-term side effects (happen within a few days or weeks), serious long-term side effects (happen within months or years), most likely long-term side effects (happen within months or years), number of medications the child is taking, possible interactions among medications, the child's discomfort or anxiety about taking a medication, and the partner's discomfort or anxiety about giving the child medication.

\section{Parent proxy reports of PROs concerning child's HRQOL, disease and treatment burden}

HRQOL was measured using parent proxy reports of the Pediatric Quality of Life (PedsQL) ${ }^{\mathrm{mm}} 4.0$ Generic Core Scales [22]. The PedsQL, including by parent proxy report, has been found to be a valid and reliable tool in pediatric rheumatology [23]. This 23-item (21 for toddlers) measure asks questions to parent proxies about their child's physical, emotional, social, and school functioning in the past month. Psychosocial and physical HRQOL summary scores and a total scale score were computed using standardized scoring [24]. Each score ranged from 0 to 100 , with higher scores indicating better HRQOL, and a score of $<78.6$ indicating suboptimal HRQOL [5].

Pain interference with aspects of daily life was measured using the 8-item PROMIS $^{\oplus}$ Parent Proxy Pain Interference Short Form, version 1.0. Parent proxies were asked about the frequency with which pain affected their child's cognitive, emotional, physical, and recreational aspects of daily life in the past week. Response 
options were: never, almost never, sometimes, often, almost always. A raw score total was computed and converted to a standardized T-score using a look-up table, with allowable values ranging from 38.0-78.0 [25].

Morning stiffness, a component of disease burden, was assessed with the question: "On a typical day over the past two weeks, how many minutes of morning stiffness did your child experience?" Response options were: none, 15 min or less, more than 15 min. Duration of morning stiffness was operationalized as a dichotomous variable where $\leq 15$ min was considered a criterion of clinically inactive disease as proposed by Wallace et al. [26].

To assess lifetime experience of a serious problem or side effect from treatment, parents were asked: "Has your child ever experienced a serious problem or side effect from a prescription medication?" Response options were yes/no.

To evaluate methotrexate intolerance, the 12-item Methotrexate Intolerance Severity Score (MISS) questionnaire [27] was administered to parent proxies of methotrexate users. The questionnaire asked about past month: abdominal pain, nausea, and vomiting that occurred before or after their child's methotrexate intake, abdominal pain and nausea that occurred at the thought of taking methotrexate, and behavioral complaints (restlessness, crying, irritability, refusal of methotrexate) associated with intake. Response options were: no complaints (0), mild complaints (1), moderate complaints (2), severe complaints (3). Sum scores ranged from 0 to 36 , and methotrexate intolerance was defined as a sum score of $\geq 6$ with at least one point on questions assessing anticipatory, associative, and/or behavioral symptoms [27]. Four subjects taking methotrexate were missing data on methotrexate intolerance and had values set conservatively to 0 (i.e., not methotrexate intolerant) following a sensitivity analysis in which all analyses were found to be unchanged when intolerance was set to 1 (intolerant) and 0 (not intolerant) (results not shown).

\section{Statistical analyses}

Analyses are of parent proxy reported PROs only and were conducted using $\mathrm{SAS}^{\oplus}$ 9.4 software (SAS Institute, Inc., Cary, North Carolina). Descriptive statistics were computed to characterize the study sample overall and by exposure to methotrexate. Differences in demographic and health characteristics by methotrexate exposure were compared using Wilcoxon rank-sum or Chi Square $\left(x^{2}\right)$ tests, as appropriate. Bivariate associations among demographic charateristics and HRQOL and measures of disease and treatment burden were compared using Wilcoxon rank-sum test and Chi Square $\left(x^{2}\right)$ test, as appropriate. Generalized estimating equations were used to estimate the contribution of disease and treatment burden variables on HRQOL, while adjusting for the multi-center sampling frame and patient demographics, including: age in years (measured continuously), sex, race/ethnicity, parent education, and disease duration in years (measured continously). Initial models tested associations among individual PROs and HRQOL, adjusting for demographic and clinical characteristics (i.e., disease duration). Subsequent models tested covariate-adjusted multivariate associations among measures of disease burden (both pain interference and morning stiffness) and HRQOL, and among measures of treatment burden (both serious medication side effect and methotrexate intolerance) and HRQOL; the final model simultaneously estimated the effects of all four PROs on HRQOL. Twenty-seven subjects were missing a PGA score (abtsracted from CARRA Registry) on the visit date; participants who were missing a PGA score did not differ from participants who had a PGA score on disease or treatment burden or by demographics with the exception of age (patients who had a PGA score were older on average than those missing a score). A sensitivity analysis was undertaken to ascertain whether values for associations among PROs and HRQOL changed when PGA score was included in multivariate regression models as an additional measure of disease activity.

\section{Results \\ Sample characteristics}

In the total sample, patients were predominantly white non-Hispanic $(81.1 \%)$, female $(76.7 \%)$, and on average 11.8 years of age (range $2-17, \mathrm{SD}=3.6$ ). The mean duration of disease was 7.7 years ( $\mathrm{SD}=3.5$ years). Parents rated their child's overall health favorably (mean $8.2, \mathrm{SD}=2.0)$. More than half $(64.4 \%)$ of patients were taking a non-biologic DMARD, with $50.0 \%$ of the cohort taking methotrexate (Table 1). For more than half of patients $(64.4 \%)$, routine visits to the rheumatologist occurred every 3 months. Nearly all parents (97.2\%) were "very" or "somewhat satisfied" with their child's care.

\section{Top concerns of parents around their child's treatment}

Nearly two-thirds (63.3\%) of parents indicated that serious long-term side effects of medications comprised their primary or secondary concern regarding their child's treatment (Fig. 1), while nearly half (46.1\%) of parents indicated that safety of medication was their primary or secondary concern regarding their child's treatment. Other issues, including the correct dose and scheduling of medication and serious short-term side effects of medications comprised top concern for smaller percentages of parents. 
Table 1 Demographic and health characteristics of the sample, in aggregate and by exposure to methotrexate

\begin{tabular}{|c|c|c|c|c|}
\hline & Total & MTX users & Non-MTX users & $P$ value \\
\hline & N (\%) & N (\%) & N (\%) & \\
\hline Total N & 180 & $90(50.0)$ & $90(50.0)$ & \\
\hline \multicolumn{5}{|l|}{ Demographics } \\
\hline Age in years (range 2-17), mean (SD) & $11.8(3.6)$ & $11.3(3.7)$ & $12.3(3.5)$ & 0.0572 \\
\hline Sex & & & & 0.7245 \\
\hline Female & $138(76.7)$ & $68(75.6)$ & $70(77.8)$ & \\
\hline Male & $42(23.3)$ & $22(24.4)$ & $20(22.2)$ & \\
\hline Race/Ethnicity & & & & 0.1277 \\
\hline White and non-Hispanic & $146(81.1)$ & $69(76.7)$ & 77 (85.6) & \\
\hline Other & $34(18.9)$ & $21(23.3)$ & $13(14.4)$ & \\
\hline Parent education level & & & & 0.3119 \\
\hline$\leq$ High school graduate & $48(26.7)$ & $21(23.3)$ & $27(30.0)$ & \\
\hline Any college & $132(73.3)$ & $69(76.7)$ & $63(70.0)$ & \\
\hline \multicolumn{5}{|l|}{ Clinical characteristics } \\
\hline Disease duration ${ }^{\mathrm{a}}$ in years, mean (SD) & $7.7(3.5)$ & $7.4(3.4)$ & $8.0(3.6)$ & 0.1853 \\
\hline Physician Global Assessment ${ }^{\mathrm{b}}$, mean (SD) & $0.9(1.3)$ & $0.9(1.3)$ & $1.0(1.4)$ & 0.7725 \\
\hline Overall health rating (range 1-10), mean (SD) & $8.2(2.0)$ & $8.2(2.1)$ & $8.2(2.0)$ & 0.9036 \\
\hline \multicolumn{5}{|l|}{ Current medications reported using ${ }^{c}$ : } \\
\hline Non-Biologic DMARD & $116(64.4)$ & $89(98.9)^{d}$ & $27(30.0)$ & $<0.0001$ \\
\hline Biologic DMARD & $105(58.3)$ & $52(57.8)$ & $53(58.9)$ & 0.8798 \\
\hline NSAID & $93(51.7)$ & $44(48.9)$ & $49(54.4)$ & 0.4558 \\
\hline Steroid & $29(16.1)$ & $15(16.7)$ & $14(15.6)$ & 0.8393 \\
\hline Herbals or non-vitamin supplement & $13(7.2)$ & $4(4.4)$ & $9(10.0)$ & 0.1499 \\
\hline Frequency of routine visits to rheumatologist & & & & 0.0787 \\
\hline Monthly & $10(5.6)$ & $6(6.7)$ & $4(4.4)$ & \\
\hline Every 6 to 8 weeks & $12(6.7)$ & $9(10.0)$ & $3(3.3)$ & \\
\hline Every 3 months & $116(64.4)$ & $61(67.8)$ & $55(61.1)$ & \\
\hline Every 6 months & $33(18.3)$ & $11(12.2)$ & $22(24.4)$ & \\
\hline Every 9-12 months & $9(5.0)$ & $3(3.3)$ & $6(6.7)$ & \\
\hline Satisfaction of child's medical care & & & & 0.1972 \\
\hline Very satisfied & $161(89.4)$ & $84(93.3)$ & $77(85.6)$ & \\
\hline Somewhat satisfied & $14(7.8)$ & $5(5.6)$ & $9(10.0)$ & \\
\hline Somewhat or very dissatisfied & $5(2.8)$ & $1(1.1)$ & $4(4.4)$ & \\
\hline
\end{tabular}

Column percentages are displayed

$P$-values derived from the chi-squared $\left(x^{2}\right)$ or Wilcoxon tests

MTX methotrexate, DMARDs disease-modifying antirheumatic drugs, NSAIDs nonsteroidal anti-inflammatory drugs, Steroids included joint injections, oral steroids, intravenous steroids, or steroid eye drops

${ }^{a}$ Disease duration was calculated by subtracting age of disease onset from age at survey administration date

${ }^{b}$ Physician Global Assessment results are reported based on sub-sample of $N=153$ participants who had non-missing values for this measure ( $N=78$ in the MTX users sub-sample). Physician global assessment has an allowable range of 0-10; the range in the study sample presented in Table 1 was $0-6$

${ }^{c}$ Medication ategories are not mutually exclusive, therefore, medications do not sum to $100 \%$

${ }^{d}$ Value does not equal $100 \%$ due to a participant's discrepancy in self-reporting

Disease symptoms and disease activity, treatment burden, and their associations with demographic and disease characteristics

The average PedsQL total score was 76.7 (SD = 18.2), average PedsQL psychosocial score was 76.9 (SD = 17.9), and average PedsQL physical score was 76.2
$(\mathrm{SD}=22.0) \quad$ (Table 2$)$. The average pain interference score was 50.1, and nearly one in five (17.8\%) respondents reported experiencing $>15$ min of morning stiffness on a typical day in the past 2 weeks (Table 3). In regards to medication related problems, more than one quarter (26.7\%) of participants 


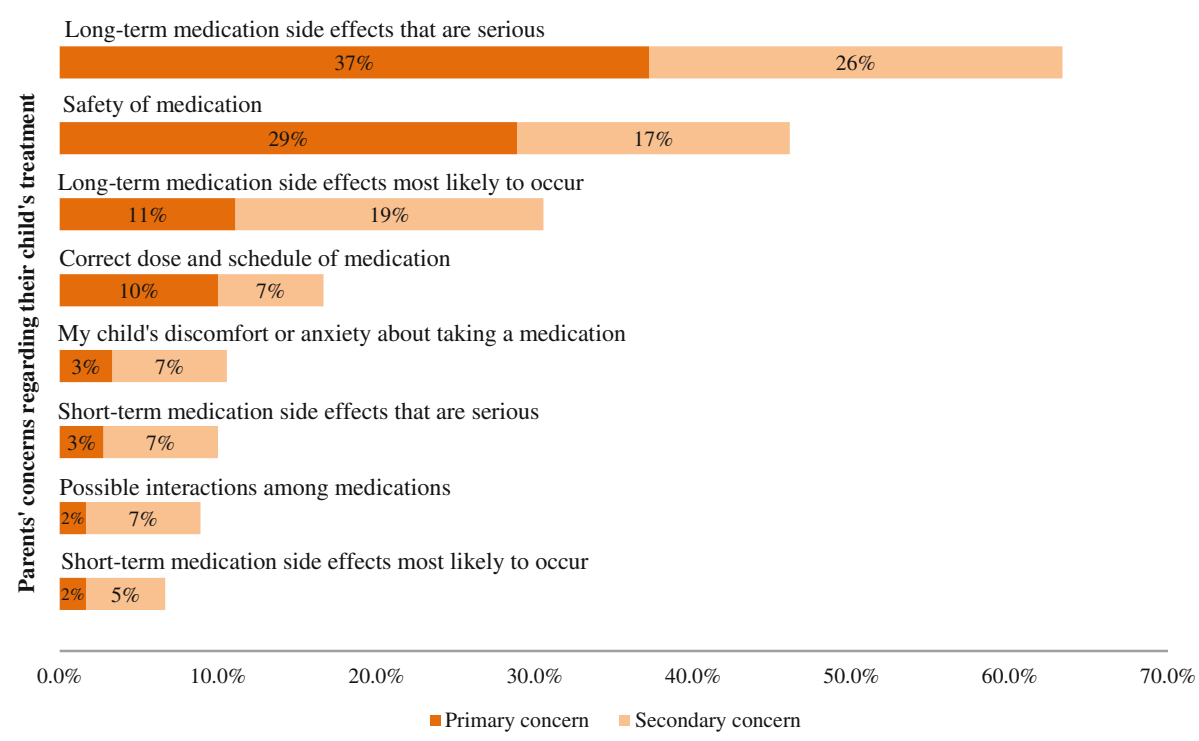

Fig. 1 Parents' top concerns regarding their child's treatment. Parents $(N=180)$ were asked to rank the primary and secondary issues that concerned them most about their child's treatment. The percentages of parents who endorsed each concern as the primary or secondary concern are presented

Table 2 Average score and demographic correlates of pediatric quality of life (PedsQL)

\begin{tabular}{|c|c|c|c|c|c|c|c|c|c|}
\hline & \multicolumn{3}{|c|}{ PedsQL total score } & \multicolumn{3}{|c|}{ PedsQL psychosocial score } & \multicolumn{3}{|c|}{ PedsQL physical score } \\
\hline & Median & Mean & SD & Median & Mean & SD & Median & Mean & SD \\
\hline Total $(N=180)$ & 81.0 & 76.7 & 18.2 & 78.3 & 76.9 & 17.9 & 81.3 & 76.2 & 22.0 \\
\hline \multicolumn{10}{|l|}{ Disease duration } \\
\hline$>8$ years & 80.4 & 76.0 & 19.4 & 80.0 & 76.8 & 18.8 & 78.1 & 74.7 & 23.5 \\
\hline$\leq 8$ years & 81.5 & 77.1 & 17.3 & 78.3 & 77.0 & 17.4 & 81.3 & 77.2 & 21.0 \\
\hline$p$-value & 0.8949 & & & 0.9514 & & & 0.5774 & & \\
\hline \multicolumn{10}{|l|}{ Age group } \\
\hline$\geq 13$ years & 79.4 & 75.4 & 18.0 & 78.3 & 76.6 & 17.0 & 75.0 & 73.1 & 22.9 \\
\hline$<13$ years & 82.6 & 77.7 & 18.4 & 78.3 & 77.2 & 18.7 & 84.4 & 78.6 & 21.1 \\
\hline$p$-value & 0.3019 & & & 0.5747 & & & 0.0878 & & \\
\hline \multicolumn{10}{|l|}{ Sex } \\
\hline Female & 79.9 & 76.2 & 18.4 & 78.3 & 76.7 & 18.1 & 76.6 & 75.3 & 22.1 \\
\hline Male & 83.2 & 78.3 & 17.5 & 80.0 & 77.8 & 17.6 & 86.6 & 79.1 & 21.7 \\
\hline$p$-value & 0.5836 & & & 0.7350 & & & 0.3559 & & \\
\hline \multicolumn{10}{|l|}{ Race/Ethnicity } \\
\hline White non-Hispanic & 82.6 & 78.0 & 17.8 & 80.0 & 77.8 & 17.7 & 84.4 & 78.2 & 21.3 \\
\hline Other & 71.2 & 71.0 & 18.9 & 75.8 & 73.1 & 18.9 & 62.5 & 67.3 & 23.1 \\
\hline$p$-value & 0.0441 & & & 0.1624 & & & 0.0110 & & \\
\hline \multicolumn{10}{|l|}{ Highest parental education } \\
\hline$\leq$ High school graduate & 82.1 & 78.6 & 17.1 & 84.2 & 80.8 & 16.1 & 78.1 & 74.5 & 22.5 \\
\hline Any college & 79.9 & 76.0 & 18.6 & 77.6 & 75.5 & 18.4 & 81.3 & 76.8 & 21.9 \\
\hline$p$-value & 0.4393 & & & 0.0911 & & & 0.5409 & & \\
\hline
\end{tabular}

Data are presented as median, mean and standard deviation (SD) of PedsQL scores

$P$-values derived from the chi-squared $\left(\mathrm{X}^{2}\right)$ or Wilcoxon tests

PedsQL Pediatric Quality of Life Inventory 4.0. Higher score means better quality of life. Possible range of PedsQL scores is from 0 to 100

Disease duration was dichotomized using the sample mean rounded to the nearest integer as the cut point 
Table 3 Prevalence and demographic correlates of disease burden and treatment burden

\begin{tabular}{|c|c|c|c|c|}
\hline & \multicolumn{2}{|l|}{ Disease burden } & \multicolumn{2}{|l|}{ Treatment burden } \\
\hline & Pain interference T-score ${ }^{a}$ & Morning stiffness & Serious medication side effect & Intolerance to methotrexate ${ }^{b}$ \\
\hline & Mean (SD) & $\%>15$ mins & $\%$ Yes & $\%$ Intolerant \\
\hline Total $(N=180)$ & $50.1(11.1)$ & $17.8 \%$ & $26.7 \%$ & $42.2 \%$ \\
\hline \multicolumn{5}{|l|}{ Disease duration } \\
\hline$>8$ years & $50.3(11.8)$ & $24.0 \%$ & $32.0 \%$ & $41.9 \%$ \\
\hline$\leq 8$ years & $49.9(10.6)$ & $13.3 \%$ & $22.9 \%$ & $42.4 \%$ \\
\hline$p$-value & 0.8676 & 0.0650 & 0.1715 & 0.9682 \\
\hline \multicolumn{5}{|l|}{ Age group } \\
\hline$\geq 13$ years & $51.3(11.7)$ & $25.3 \%$ & $39.2 \%$ & $40.0 \%$ \\
\hline$<13$ years & $49.1(10.5)$ & $11.9 \%$ & $16.8 \%$ & $43.6 \%$ \\
\hline$p$-value & 0.1481 & 0.0193 & 0.0007 & 0.7335 \\
\hline \multicolumn{5}{|l|}{ Sex } \\
\hline Female & $50.3(11.1)$ & $18.1 \%$ & $26.1 \%$ & $48.5 \%$ \\
\hline Male & $49.3(11.1)$ & $16.7 \%$ & $28.6 \%$ & $22.7 \%$ \\
\hline$p$-value & 0.5835 & 0.8297 & 0.7499 & 0.0332 \\
\hline \multicolumn{5}{|l|}{ Race/Ethnicity } \\
\hline White non-Hispanic & $49.2(10.7)$ & $19.2 \%$ & $26.7 \%$ & $40.6 \%$ \\
\hline Other & $53.8(12.0)$ & $11.8 \%$ & $26.5 \%$ & $47.6 \%$ \\
\hline$p$-value & 0.0266 & 0.3086 & 0.9771 & 0.5674 \\
\hline \multicolumn{5}{|l|}{ Highest parental education } \\
\hline$\leq$ High school graduate & $50.2(10.6)$ & $22.9 \%$ & $27.1 \%$ & $42.9 \%$ \\
\hline Any college & $50.0(11.3)$ & $15.9 \%$ & $26.5 \%$ & $42.0 \%$ \\
\hline$p$-value & 0.7779 & 0.2768 & 0.9392 & 0.9464 \\
\hline
\end{tabular}

Data are presented as mean and standard deviation (SD) for pain interference and as row percentages for other outcomes

$P$-values derived from the chi-squared $\left(x_{2}\right)$ or Wilcoxon tests

Disease duration was dichotomized using the sample mean rounded to the nearest integer as the cut point

${ }^{a}$ Raw pain interference scores were transformed into a 'T-score' for each participant. The T-score rescales the raw score into a standardized score with a mean of

50 , standard deviation of 10 , and possible range of 38-78

${ }^{\mathrm{b}}$ Intolerance to methotrexate was assessed only among those who were on methotrexate $(N=90)$

reported that they had ever experienced at least one serious medication side effect.

Patient demographic characteristics were not associated with PedsQL scores, with the exception of race/ethnicity: on average, white non-Hispanics had higher PedsQL total scores (Mean $=78.0)$ and PedsQL physical scores $($ Mean $=78.2)$ compared to minorities $($ Mean $=71.0$, 67.3 , respectively) ( $p=0.036$ ) (Table 2 ).

Differences in pain interference score were seen between racial/ethnic group, with white non-Hispanics averaging a lower pain interference score $($ Mean $=49.2)$ than their minority counterparts (Mean $=53.8)(p=$ 0.0266). Differences in morning stiffness were seen by age, with the percentage of children experiencing $>15$ min of morning stiffness on a typical day in the past 2 weeks higher in children who were $\geq 13$ years $(25.3 \%)$ compared to younger than 13 years $(11.9 \%, p=0.0193)$. Similarly, $39.2 \%$ of children $\geq 13$ years of age reported at least one serious medication side effect compared to $16.8 \%$ of those younger than 13 years $(p=0.0007)$. Proportionately more females than males met criteria for methotrexate intolerance $(48.5 \%$ and $22.7 \%$, respectively) $(p=0.0332)$. For the 153 participants with a PGA score, the highest score reported was 6 out of a possible 10, with more than half (52.9\%) having a PGA score of 0. PGA score was not associated with demographic characteristics (Additional file 1: Table S2).

\section{Associations between disease symptoms and disease activity, treatment burden, and pediatric quality of life} In bivariate models (Table 4, Models 1a-1d), greater pain interference, $>15 \mathrm{~min}$ of morning stiffness, and having serious medication side effects were independently associated with lower total, psychosocial, and physical PedsQL scores (all $p$-values $<0.01$ ); methotrexate intolerance was associated with lower total and psychosocial PedsQL scores (all $p$-values $\leq 0.01$ ). Being on methotrexate without experiencing intolerance was associated with higher total, psychosocial, and physical PedsQL scores (all $p$-values $<0.05$ ). 
Table 4 Multivariate regression analyses measuring associations among pediatric quality of life (PedsQL) and disease activity/ symptoms and treatment burden $(N=180)$

\begin{tabular}{|c|c|c|c|c|c|c|}
\hline & \multicolumn{2}{|c|}{ PedsQL total score } & \multicolumn{2}{|c|}{ PedsQL psychosocial score } & \multicolumn{2}{|c|}{ PedsQL physical score } \\
\hline & $\beta$ (S.E.) & $p$-value & $\beta$ (S.E.) & $p$-value & $\beta$ (S.E.) & $p$-value \\
\hline \multicolumn{7}{|l|}{ Model 1a-1d Individual PROs } \\
\hline 1a) Pain interference ${ }^{a}$ & $-1.30(0.05)$ & $<0.0001$ & $-1.17(0.04)$ & $<0.0001$ & $-1.53(0.10)$ & $<0.0001$ \\
\hline \multicolumn{7}{|l|}{ 1b) Morning stiffness } \\
\hline$>15 \min$ & $-19.92(0.91)$ & $<0.0001$ & $-16.77(1.23)$ & $<0.0001$ & $-25.65(2.05)$ & $<0.0001$ \\
\hline$\leq 15 \min$ & reference & & reference & & reference & \\
\hline \multicolumn{7}{|l|}{ 1c) Serious medication side effect } \\
\hline Any & $-12.54(3.77)$ & 0.0009 & $-10.82(3.54)$ & 0.0023 & $-15.68(4.75)$ & 0.0010 \\
\hline None & reference & & reference & & reference & \\
\hline \multicolumn{7}{|l|}{ 1d) Methotrexate status } \\
\hline With methotrexate intolerance & $-3.66(1.42)$ & 0.0100 & $-3.96(0.77)$ & $<0.0001$ & $-3.15(3.20)$ & 0.3239 \\
\hline On methotrexate without intolerance & $4.89(1.24)$ & $<0.0001$ & $4.84(1.43)$ & 0.0007 & $4.91(2.49)$ & 0.0487 \\
\hline Not on methotrexate & reference & & reference & & reference & \\
\hline \multicolumn{7}{|l|}{ Model 2 Disease Burden PROs } \\
\hline Pain interference ${ }^{a}$ & $-1.27(0.05)$ & $<0.0001$ & $-1.17(0.05)$ & $<0.0001$ & $-1.45(0.09)$ & $<0.0001$ \\
\hline \multicolumn{7}{|l|}{ Morning stiffness } \\
\hline$>15 \min$ & $-1.99(0.50)$ & $<0.0001$ & $-0.28(0.92)$ & 0.7611 & $-5.13(1.81)$ & 0.0046 \\
\hline$\leq 15 \min$ & reference & & reference & & reference & \\
\hline \multicolumn{7}{|l|}{ Model 3 Treatment Burden PROs } \\
\hline \multicolumn{7}{|l|}{ Serious medication side effect } \\
\hline Any & $-11.68(3.77)$ & 0.0020 & $-9.87(3.70)$ & 0.0076 & $-15.10(4.56)$ & 0.0009 \\
\hline None & reference & & reference & & reference & \\
\hline \multicolumn{7}{|l|}{ Methotrexate status } \\
\hline With methotrexate intolerance & $-2.13(1.34)$ & 0.1121 & $-2.66(0.30)$ & $<0.0001$ & $-1.15(3.46)$ & 0.7406 \\
\hline On methotrexate without intolerance & $3.89(1.64)$ & 0.0175 & $4.01(2.14)$ & 0.0607 & $3.73(1.67)$ & 0.0257 \\
\hline Not on methotrexate & reference & & reference & & reference & \\
\hline \multicolumn{7}{|l|}{ Model 4 All PROs } \\
\hline Pain interference ${ }^{a}$ & $-1.21(0.07)$ & $<0.0001$ & $-1.12(0.07)$ & $<0.0001$ & $-1.38(0.08)$ & $<0.0001$ \\
\hline \multicolumn{7}{|l|}{ Morning stiffness } \\
\hline$>15 \min$ & $-2.39(0.17)$ & $<0.0001$ & $-0.73(0.80)$ & 0.3628 & $-5.48(1.88)$ & 0.0035 \\
\hline$\leq 15 \min$ & reference & & reference & & reference & \\
\hline \multicolumn{7}{|l|}{ Serious medication side effect } \\
\hline Any & $-3.64(1.89)$ & 0.0548 & $-2.67(2.18)$ & 0.2201 & $-5.47(2.28)$ & 0.0163 \\
\hline None & reference & & reference & & reference & \\
\hline \multicolumn{7}{|l|}{ Methotrexate status } \\
\hline With methotrexate intolerance & $-3.24(0.92)$ & 0.0004 & $-3.57(1.06)$ & 0.0008 & $-2.55(2.48)$ & 0.3031 \\
\hline On methotrexate without intolerance & $1.47(0.39)$ & 0.0001 & $1.83(0.73)$ & 0.0119 & $0.94(2.11)$ & 0.6540 \\
\hline Not on methotrexate & reference & & reference & & reference & \\
\hline
\end{tabular}

All estimates were obtained using generalized estimating equations (GEE) to account for clustering within clinics and were adjusted for demographics including age (continuous), sex, race/ethnicity, parent education, and disease duration (continuous); regression coefficients ( $\beta$ ) and their standard errors (SE) are presented Model 1a-1d assessed association between PedsQL score and each individual PRO

Model 2 assessed association between PedsQL score and both of the disease burden PROs

Model 3 assessed association between PedsQL score and both of the treatment burden PROs

Model 4 included all PROs simultaneously

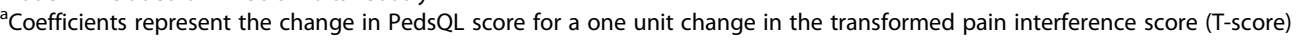


Controlling for both disease burden PROs simultaneously (Table 4, Model 2), partly attenuated the relationsip between these PROs and PedsQL scores; the effect of morning stiffness on the PedsQL psychosocial score was no longer significant after adjustment for pain interference. Controlling for both treatment burden PROs simultaneously (Table 4, Model 3) partly attenuated the relationships between these PROs and PedsQL scores; the effect of methotrexate intolerance on the PedsQL total and physical scores was no longer significant after adjustment for having any serious medication side effects. Simultaneous adjustment for all PROs (Table 4, Model 4) revealed further attenuation yet significant effects on PedsQL total score. Notably, only pain interference and methotrexate status remained significantly associated with PedsQL psychosocial score (all $p$-values $<0.05$ ), while pain interference, morning stiffness and serious medication side effects were significantly associated with PedsQL physical score (all $p$-values $<0.05$ ). Analysis restricted to participants with a value for PGA did not change our findings when PGA was added into models 2 and 4 as a covariate (Additional file 1: Table S3).

\section{Discussion}

We engaged parents of children/adolescents with JIA in providing structured PROs to describe their child's experiences of their condition and its treatment, to augment clinical data collected in a Registry and inform understanding of HRQOL. We leveraged a scalable disease Registry constructed from a novel informatics architecture to accomplish this goal, creating a channel for representation in the Registry of the "patient voice." Parents were highly willing to engage; nearly $70 \%$ of those approached consented to provide reports about their concerns, and their child's disease and treatment experiences.

Parents' top concerns centered on medication safety and treatment side effects - especially notable in relation to potential for long-term harm stemming from treatment. While data from the Registry and The Learning Cohort cannot yet prospectively characterize longterm safety issues arising from rheumatic disease treatment, lifetime experiences of problems or side effects from medications and intolerance to methotrexate were measured. More than one-quarter of the cohort reported experiencing a serious problem or side effect from a prescription medication and nearly half of the cohort on methotrexate reported intolerance. Adverse treatment experiences negatively impacted HRQOL for children/ adolescents with JIA, consistent with other reports [28].

In our study cohort, HRQOL, disease experience, and treatment burden varied by patient demographic characteristics, although no systematic pattern emerged. Relative to established thresholds [5] average HRQOL among the cohort was suboptimal. Non-white-and/or Hispanic youth had lower levels of physical HRQOL than did white non-Hispanic youth. Higher prevalence of experiencing $>15$ min of morning stiffness was seen among older youth who also reported a higher prevalence of experiencing a serious side effect or problem from their medication. Among those taking methotrexate, a greater proportion of females compared to males reported symptoms of intolerance.

Measures of disease and treatment burden were negatively associated with HRQOL as expected. Importantly, as hypothesized, measures of treatment burden were negatively associated with HRQOL even after controlling for demographic characteristics, clinical measures of disease duration and disease activity, and patientreported measures of disease burden. While treatment has improved for JIA [29], further work is needed. Treatment side effects and problems have potential to undermine adherence $[28,30]$, which may negatively impact disease management and outcomes [31]; treatment may be stressful, further reducing HRQOL. The strong negative association between methotrexate intolerance and PedsQL psychosocial score point to the emotionally burdensome aspect of treatment problems, consistent with reports about the negative effects on HRQOL of perceived treatment burden [6]. Evaluation of therapies in the context of their acceptability and a broad calculus of health and wellbeing is warranted with attention paid to modifying treatment regimens and improving therapeutics. Strategies for better educating patients about these issures merit attention, and research to identify effective approaches to educate patients and their parents to the potential that treatment side effects may arise which may undermine wellbeing so that early ameliorative action can be taken to optimize benefits and minimize harms.

This report supports a growing literature on the experience of HRQOL for youth with JIA that considers the combined effects of disease and treatment burden. It also provides a proof of concept for prospective collection of PROs to augment clinical registry data collection to foster a virtuous cycle of cohort engagement and "patient voice," a model that could drive CER using a PCOR orientation. Understanding threats to HRQOL - including those related to treatment - is vital to improving care of youth with JIA. Further research is needed to more clearly specify and ameliorate the negative effects of treatment on wellbeing, considering the potential for problems to reflect burdens related to sensorial (e.g., pain), psychosocial (e.g., fear), and physiologic (e.g., rash) aspects of treatment - which may require different responses.

Strengths of this report include use of validated structured PROs among a registry enrolled cohort with 
confirmed diagnosis. Nevertheless several limitations merit discussion. Data were collected from several geographically diverse clinics, however findings are not generalizable to all youth with JIA. This report reflects parent proxy data only. Parent proxy reports may differ from child report [32-35]. The measure of serious side effects reflects lifetime experience and does not afford a view into the effects on HRQOL of temporally proximal versus distal harms; nevertheless, for those on methotrexate, intolerance is measured for a time period that directly relates to study outcomes. Finally, all selfreported and retrospectively reported data are subject to reporting bias.

\section{Conclusions}

Engaging patients and their parent proxies as partners in research agenda setting and reporting about disease and treatment experiences yields a rich, informative view into HRQOL for children/adolescents with JIA. This view highlights the importance of evaluating both treatment experiences and disease burden when measuring outcomes. This model may help build an evidence base toward improved treatment of JIA and provides a proof of concept for integrating PROs into clinical registries a model with high translation potential to other pediatric onset chronic diseases. A nuanced picture of benefit and harm may enable development of better therapies and supportive interventions, consistent with forward looking models for PCOR and CER and driving goals for helping patients with chronic illness "live well" [36].

\section{Additional file}

Additional file 1: Table S1. Primary Measures and Scoring. Table S2. Association between demographic characteristics and Physician Global Assessment (PGA) score among a sample restricted to participants with a value for PGA score $(N=153)$. Table S3. Multivariate regression analyses measuring associations among pediatric quality of life (PedsQL) and disease activity/symptoms and treatment burden among a sample restricted to participants with a value for Physician Global Assessment (PGA) (N=153). (DOCX $60 \mathrm{~kb})$

\section{Abbreviations}

CARRA: Childhood Arthritis and Rheumatology Research Alliance; CER: Comparative effectiveness research; DMARD: Disease modifying antirheumatic drug; HRQOL: Health-related quality of life; JIA: Juvenile Idiopathic Arthritis; MISS: Methotrexate Intolerance Severity Score; NSAID: Nonsteroidal anti-inflammatory drug; PCOR: Patient-centered outcomes research; PedsQL: Pediatric Quality of Life; PGA: Physician Global Assessment; PROs: Patient Reported Outcomes; SD: Standard Deviation; TLC: The Learning Cohort

\section{Acknowledgements}

We gratefully acknowledge the Childhood Arthritis and Rheumatology Research Alliance (CARRA) Registry for providing access to study sites and subjects and enabling this research. Thoughtful guidance on the measurement battery was obtained from Drs. Laura Schanberg, Esi Morgan, and Christy Sandborg. Statistical programming was conducted by Qian Huang and Joseph Allario.

\section{Funding}

This study was funded by grants from the National Library of Medicine $(\mathrm{NIH})$ award number R01LM011185 and the Patient-Centered Outcomes Research Institute (PCORI) award number CDRN130604608. The funding bodies (NIH, PCORI) were not involved in the design of the study and collection, analysis, and interpretation of data, nor in writing the manuscript.

\section{Availability of data and materials}

Data will not be shared. Data used in this report are clinical data, and authors have an ethical and legal responsibility to respect participants' rights to privacy and to protect their identity. Raw data used for this report are identifiable. Participants were not consented with the expectation that their raw data would be made widely available, rather, participants were consented under the expectation that their data and study results would be reported in aggregate form only.

\section{Authors' contributions}

ERW conceptualized and designed the study, designed data collection measures, and protocols, supervised data collection, directed data anlyses, and drafted the initial manuscript. LEW advised on data analysis and and interpretation of data and results, and assisted with drafting and revising the manuscript. PKS performed data collection, and assisted with the drafting and revising of the manuscript. KMM contributed to data analysis and interpretation of data and results, and assisted with drafting and revising the manuscript. FD assisted in the conception and design of the study, advised on data collection measures, and critically reviewed the manuscript for intellectual content. $\mathrm{AOH}$ assisted in the conception and design of the study, advised on data collection measures, and critically reviewed the manuscript for intellectual content. YK assisted in the conception and design of the study, advised on data collection measures, and critically reviewed the manuscript for intellectual content. KDM assisted in the conception and design of the study, advised on data collection measures, and critically reviewed the manuscript for intellectual content. SR assisted in the conception and design of the study, advised on data collection measures, and critically reviewed the manuscript for intellectual content. MN assisted in the conception and design of the study, advised on data collection measures, assisted with data collection, and critically reviewed the manuscript for intellectual content. All authors approved the final manuscript as submitted. All authors agree to be accountable for all aspects of the work in ensuring that questions related to the accuracy or integrity of any part of the work are appropriately investigated and resolved.

\section{Ethics approval and consent to participate}

The Institutional Review Boards of each of the four participating sites (Boston Children's Hospital, Seattle Children's Hospital, Joseph M. Sanzari Children's Hospital at Hackensack University Medical Center, and University of Utah/ Primary Children's Hospital) approved the study. Written informed consent/ assent was obtained from all parent/child participants included in the study.

Consent for publication

Not applicable

\section{Competing interests}

The authors declare that they have no competing interests.

\section{Publisher's Note}

Springer Nature remains neutral with regard to jurisdictional claims in published maps and institutional affiliations.

\section{Author details}

${ }^{1}$ Division of Adolescent/Young Adult Medicine, Boston Children's Hospital, 300 Longwood Ave, Boston, MA 02115, USA. ²Department of Pediatrics, Harvard Medical School, Boston 02115, USA. ${ }^{3}$ Computational Health Informatics Program, Boston Children's Hospital, 300 Longwood Ave, Boston, MA 02115, USA. Division of Developmental Medicine, Boston Children's Hospital, 300 Longwood Ave, Boston, MA 02115, USA. ${ }^{5}$ Rheumatology Program, Division of Immunology, Boston Children's Hospital, 300 Longwood Ave, Boston, MA 02115, USA. ${ }^{6}$ Division of Pediatric Rheumatology, University of Utah School of Medicine and Primary Children's Medical Center, Salt Lake City, UT 84113, USA. 7 Division of Pediatric Rheumatology, Hackensack University Medical Center, Hackensack, NJ 07601, USA. ${ }^{8}$ Department of Biomedical Informatics, Harvard Medical School, Boston 02115, USA. 'Division of Rheumatology, Seattle Children's Hospital, Seattle, WA 98105, USA. 
Received: 28 August 2017 Accepted: 14 December 2017

\section{Published online: 04 January 2018}

\section{References}

1. Wallace, C. A., Huang, B., Bandeira, M., Ravelli, A., \& Giannini, E. H. (2005). Patterns of clinical remission in select categories of juvenile idiopathic arthritis. Arthritis Rheum, 52(11), 3554-3562. https://doi.org/10.1002/art.21389.

2. Ringold, S., Seidel, K. D., Koepsell, T. D., \& Wallace, C. A. (2009). Inactive disease in polyarticular juvenile idiopathic arthritis: Current patterns and associations. Rheumatology (Oxford), 48(8), 972-977. https://doi.org/10.1093/ rheumatology/kep144.

3. Magni-Manzoni, S., Pistorio, A., Labo, E., Viola, S., Garcia-Munitis, P., Panigada, S., et al. (2008). A longitudinal analysis of physical functional disability over the course of juvenile idiopathic arthritis. Ann Rheum Dis, 67(8), 1159-1164. https://doi.org/10.1136/ard.2007.078121.

4. Gutierrez-Suarez, R., Pistorio, A., Cespedes Cruz, A., Norambuena, X., Flato, B., Rumba, l., et al. (2007). Health-related quality of life of patients with juvenile idiopathic arthritis coming from 3 different geographic areas. The PRINTO multinational quality of life cohort study. Rheumatology (Oxford), 46(2), 314-320. https://doi.org/10.1093/rheumatology/kel218.

5. Seid, M., Opipari, L., Huang, B., Brunner, H. I., \& Lovell, D. J. (2009). Disease control and health-related quality of life in juvenile idiopathic arthritis. Arthritis Rheum, 61(3), 393-399. https://doi.org/10.1002/art.24477.

6. Haverman, L., Grootenhuis, M. A., van den Berg, J. M., van Veenendaal, M., Dolman, K. M., Swart, J. F., et al. (2012). Predictors of health-related quality of life in children and adolescents with juvenile idiopathic arthritis: Results from a web-based survey. Arthritis Care Res (Hoboken), 64(5), 694-703. https://doi.org/10.1002/acr.21609.

7. Minden, K., Niewerth, M., Listing, J., Biedermann, T., Bollow, M., Schontube, M., et al. (2002). Long-term outcome in patients with juvenile idiopathic arthritis. Arthritis Rheum, 46(9), 2392-2401.

8. Oen, K., Malleson, P. N., Cabral, D. A., Rosenberg, A. M., Petty, R. E., \& Cheang, M. (2002). Disease course and outcome of juvenile rheumatoid arthritis in a multicenter cohort. J Rheumatol, 29(9), 1989-1999.

9. Packham, J. C., Hall, M. A., \& Pimm, T. J. (2002). Long-term follow-up of 246 adults with juvenile idiopathic arthritis: Predictive factors for mood and pain. Rheumatology (Oxford), 41(12), 1444-1449.

10. Zak, M., \& Pedersen, F. K. (2000). Juvenile chronic arthritis into adulthood: A long-term follow-up study. Rheumatology (Oxford), 39(2), 198-204.

11. Luca, N. J., \& Feldman, B. M. (2014). Health outcomes of pediatric rheumatic diseases. Best Pract Res Clin Rheumatol, 28(2), 331-350. https://doi.org/10. 1016/j.berh.2014.04.001.

12. Lavallee, D. C., Chenok, K. E., Love, R. M., Petersen, C., Holve, E., Segal, C. D., et al. (2016). Incorporating patient-reported outcomes into health care to engage patients and enhance care. Health Aff (Millwood), 35(4), 575-582. https://doi.org/10.1377/hlthaff.2015.1362.

13. Kamper, S. J., Dissing, K. B., \& Hestbaek, L. (2016). Whose pain is it anyway? Comparability of pain reports from children and their parents. [journal article] Chiropr Man Ther, 24(1), 24. https:/doi.org/10.1186/s12998-016-0104-0.

14. Brudvik, C., Moutte, S. D., Baste, V., \& Morken, T. (2016). A comparison of pain assessment by physicians, parents and children in an outpatient setting. Emerg Med J. https://doi.org/10.1136/emermed-2016-205825.

15. Woodward, L., Johnson, S., Walle, J. V., Beck, J., Gasteyger, C., Licht, C., et al. (2016). An innovative and collaborative partnership between patients with rare disease and industry-supported registries: The global aHUS registry. Orphanet J Rare Dis, 11(1), 154. https://doi.org/10.1186/s13023-016-0537-5.

16. Javaid, M. K., Forestier-Zhang, L., Watts, L., Turner, A., Ponte, C., Teare, H., et al. (2016). The RUDY study platform - a novel approach to patient driven research in rare musculoskeletal diseases. Orphanet J Rare Dis, 11(1), 150. https://doi.org/10.1186/s13023-016-0528-6.

17. Natter, M. D., Quan, J., Ortiz, D. M., Bousvaros, A., Ilowite, N. T., Inman, C. J., et al. (2013). An i2b2-based, generalizable, open source, self-scaling chronic disease registry. J Am Med Inform Assoc, 20(1), 172-179. https://doi.org/10. 1136/amiajnl-2012-001042

18. Robinson, A. B., Hoeltzel, M. F., Wahezi, D. M., Becker, M. L., Kessler, E. A., Schmeling, H., et al. (2014). Clinical characteristics of children with juvenile dermatomyositis: The childhood arthritis and rheumatology research alliance registry. Arthritis Care Res (Hoboken), 66(3), 404-410. https://doi.org/10.1002/acr.22142.

19. Ringold, S., Beukelman, T., Nigrovic, P. A., \& Kimura, Y. (2013). Race, ethnicity, and disease outcomes in juvenile idiopathic arthritis: A cross-sectional analysis of the childhood arthritis and rheumatology research alliance
(CARRA) registry. J Rheumatol, 40(6), 936-942. https://doi.org/10.3899/ jrheum.121147.

20. Harris, P. A., Taylor, R., Thielke, R., Payne, J., Gonzalez, N., \& Conde, J. G. (2009). Research electronic data capture (REDCap) - a metadata-driven methodology and workflow process for providing translational research informatics support. J Biomed Inform, 42(2), 377-381. https://doi.org/10. 1016/j.jbi.2008.08.010.

21. Taxter, A. J., Wileyto, E. P., Behrens, E. M., \& Weiss, P. F. (2015). Patient reported outcomes across categories of juvenile idiopathic arthritis. $J$ Rheumatol, 42(10), 1914-1921. https://doi.org/10.3899/jrheum.150092.

22. Varni, J. W. (2016). The PedsQL measurement model for the pediatric quality of life inventory. http://www.pedsql.org/about_pedsql.html.

23. Varni, J. W., Seid, M., Smith Knight, T., Burwinkle, T., Brown, J., \& Szer, I. S. (2002). The PedsQL in pediatric rheumatology: Reliability, validity, and responsiveness of the pediatric quality of life inventory generic Core scales and rheumatology module. Arthritis Rheum, 46(3), 714-725.

24. Varni, J. W. (2014). Scaling and scoring of the pediatric quality of life inventory PedsQL. France: Lyon.

25. A brief guide to the PROMIS Pain interference instruments (2015). PatientReported Outcomes Measurement Inforamtion System.

26. Wallace, C. A., Giannini, E. H., Huang, B., Itert, L., \& Ruperto, N. (2011). American College of Rheumatology provisional criteria for defining clinical inactive disease in select categories of juvenile idiopathic arthritis. Arthritis Care Res (Hoboken), 63(7), 929-936. https://doi.org/10.1002/acr.20497.

27. Bulatovic, M., Heijstek, M. W., Verkaaik, M., van Dijkhuizen, E. H., Armbrust, W., Hoppenreijs, E. P., et al. (2011). High prevalence of methotrexate intolerance in juvenile idiopathic arthritis: Development and validation of a methotrexate intolerance severity score. Arthritis Rheum, 63(7), 2007-2013. https://doi.org/10.1002/art.30367.

28. Mulligan, K., Wedderburn, L. R., \& Newman, S. (2015). The experience of taking methotrexate for juvenile idiopathic arthritis: Results of a crosssectional survey with children and young people. Pediatr Rheumatol Online J, 13, 58. https://doi.org/10.1186/s12969-015-0052-6.

29. Blazina, S., Markelj, G., Avramovic, M. Z., Toplak, N., \& Avcin, T. (2016). Management of Juvenile Idiopathic Arthritis: A clinical guide. Paediatr Drugs, 18(6), 397-412. https://doi.org/10.1007/s40272-016-0186-0.

30. Mulligan, K., Kassoumeri, L., Etheridge, A., Moncrieffe, H., Wedderburn, L. R., \& Newman, S. (2013). Mothers' reports of the difficulties that their children experience in taking methotrexate for juvenile idiopathic arthritis and how these impact on quality of life. Pediatr Rheumatol Online J, 11(1), 23. https:// doi.org/10.1186/1546-0096-11-23.

31. Foster, H. E., Marshall, N., Myers, A., Dunkley, P., \& Griffiths, I. D. (2003). Outcome in adults with juvenile idiopathic arthritis: A quality of life study. Arthritis Rheum, 48(3), 767-775. https://doi.org/10.1002/art.10863.

32. Vanoni, F., Suris, J. C., von Scheven-Gete, A., Fonjallaz, B., \& Hofer, M. (2016). The difference of disease perception by juvenile idiopathic arthritis patients and their parents: Analysis of the JAMAR questionnaire. Pediatr Rheumatol Online J, 14(1), 2. https://doi.org/10.1186/s12969-015-0063-3.

33. Janse, A. J., Uiterwaal, C. S., Gemke, R. J., Kimpen, J. L., \& Sinnema, G. (2005). A difference in perception of quality of life in chronically ill children was found between parents and pediatricians. J Clin Epidemiol, 58(5), 495-502. https://doi.org/10.1016/j.jclinepi.2004.09.010.

34. Lal, S. D., McDonagh, J., Baildam, E., Wedderburn, L. R., Gardner-Medwin, J., Foster, H. E., et al. (2011). Agreement between proxy and adolescent assessment of disability, pain, and well-being in juvenile idiopathic arthritis. J Pediatr, 158(2), 307-312. https://doi.org/10.1016/j.jpeds.2010.08.003.

35. Vetter, T. R., Bridgewater, C. L., \& McGwin Jr., G. (2012). An observational study of patient versus parental perceptions of health-related quality of life in children and adolescents with a chronic pain condition: Who should the clinician believe? Health Qual Life Outcomes, 10, 85. https://doi.org/10.1186/ 1477-7525-10-85.

36. Living well with chronic illness: A call for public health action (2012). Washington: Committee on Living Well with Chronic Disease: Public Action to Reduce Disability and Improve Functioning and Quality of Life; Institute of Medicine. 